\title{
Strategic Interaction between Operators in the Context of Spectrum Sharing for $5 \mathrm{G}$ Networks
}

\author{
Erwin J. Sacoto-Cabrera ${ }^{D},{ }^{1,2}$ Angel Sanchis-Cano ${ }^{(D)},{ }^{1}$ Luis Guijarro ${ }^{D},{ }^{1}$ \\ José Ramón Vidal $\mathbb{C}^{1},{ }^{1}$ and Vicent Pla $\mathbb{C}^{1}$ \\ ${ }^{1}$ ITACA, Universitat Politècnica de València, Camino de Vera s/n, 46022 Valencia, Spain \\ ${ }^{2}$ GIHP4C, Universidad Politécnica Salesiana-Sede Cuenca, Calle Vieja 12-30 y Elia Liut, 010102 Cuenca, Ecuador \\ Correspondence should be addressed to Erwin J. Sacoto-Cabrera; esacoto@ups.edu.ec
}

Received 30 May 2018; Revised 13 September 2018; Accepted 26 September 2018; Published 11 October 2018

Guest Editor: Ivan Marsa-Maestre

Copyright (C) 2018 Erwin J. Sacoto-Cabrera et al. This is an open access article distributed under the Creative Commons Attribution License, which permits unrestricted use, distribution, and reproduction in any medium, provided the original work is properly cited.

5G networks will make network sharing agreements between mobile operators technically possible. However, depending on the agreed and implemented quality-of-service isolation, the provision of services may lead to unsustainable business cases. In this paper, the economic feasibility of such arrangements is analyzed for the case of two operators. Concretely, while one network operator owns the spectrum, one virtual operator does not, and each one provides service to its subscriber base. Two sharing alternatives, namely, pooling and priority sharing, are studied regarding the profits that each operator gets. We conclude that the network operator is worse off under any circumstances under a pooling agreement, while a lump sum payment may leave the network operator better off under a priority sharing agreement.

\section{Introduction}

The fifth generation $(5 \mathrm{G})$ wireless networks will appear in the market in 2020 and is expected to improve customers' quality of service (QoS) significantly with the increasing of data volume in mobile networks [1]. Furthermore, 5G networks will have the capacity to provide different QoS to users with a wide range of needs, such as new applications, different traffic types, and a wide range of services $[2,3]$.

The sharing of any sort of resources among network operators has been shown as an efficient way of spreading out the technology at a lower cost. The resources in this paper will be typically spectrum that is owned by the network operator, but similar analysis may be performed when the resources are, e.g., radio-access-network (RAN), network roaming, and core-network $[4,5]$. We will refer to the sharing of resources with the general term network sharing. Authors of [6] describe a scenario where infrastructure providers enable the leasing of resources, which allow service providers to share the cost of providing coverage with virtual operators, Over-The-Top providers and industry vertical market players. However, network sharing is based on the dynamic deployment and scaling of functions in the $5 \mathrm{G}$ networks and requires a careful orchestration of resources to preserve bilateral agreements among operators, as stated by [7].

Regarding the costs involved in moving to $5 \mathrm{G}$, they can be significantly reduced through network densification and network sharing. The business models analyzing these scenarios such as [8] are usually focused on the relation between the service provider and the network operator, where their physical and logical network infrastructures are tightly coupled. There are several ways to implement network sharing, as proposed in [9]. A possible new business model could be based on the sharing of resources, where the owner of the resources and the operator of the resources are different actors.

In the literature, some researches are focused on the study of the economic feasibility of different network sharing scenarios based on elements of queuing theory, such as [10, 11], as well as microeconomics and game theory concepts [12, 13]. For instance, in the context of network sharing, in [14] the effects of infrastructure sharing and competition regulation 
on cellular network planning are analyzed. The authors of [15] analyze a network slicing scenario, based on game theory and a framework called "share-constrained proportional allocation"; the results obtained provide an effective and implementable scheme for dynamically sharing resources across slices. In [16], the authors investigate the generic instruments for addressing a spectrum sharing in $4 \mathrm{G}$ networks from a cooperative games perspective and conclude that, in most of the considered instances, the mobile network operators (MNOs) are better off building a unique shared RAN than creating subcoalitions or building individual RANs due to the combined gain from spectrum aggregation and cost reduction from sharing the network infrastructure. In [17], the authors investigate a particular class of congestion games and learning mechanisms to design a distributed solution to the wireless network slicing problem. In the same way, there are some studies that analyze the network sharing, based on game theory, such as [18-21]. In the framework of the queue theory, in [22] the effects of queueing delays and users' related costs on the management and control computing resources are analyzed. The authors of [23] propose an analysis from a pricing perspective, based on priority queuing (PQ) and Generalized Processor Sharing, with the issue of maximizing networks operators revenue. In [24], the authors investigate the priority queuing as a way to establish service differentiation; to do that, they consider the Discriminatory Processor Sharing discipline for two models of service with different QoS and determine the prices that maximize the provider's profit. In the same way, [25] studies pricing for heterogeneous services based on a priority queuing as a way to establish service differentiation. Additionally, in [26] the authors analyze the cooperation strategies among mobile network operators competitors, customers, and different types of partners based on network sharing. However, the mentioned studies do not analyze the incentives to mobile operators to engage in spectrum sharing schemes using priority sharing disciplines in a network sharing environment from an economic point of view.

Our aim in this paper is to proceed one step further and model the strategic interaction in network sharing between a network operator and a virtual operator, providing service to their users. We analyze two alternatives: pooling and priority sharing. The analysis is conducted by means of game theory. Our main contribution is to prove that network sharing is economically viable and allows the network and virtual operators to coexist under a priority sharing agreement.

The rest of this paper is organized as follows. In Section 2, we describe in detail the model with the actors, the utility of each actor, and the pricing scheme. In Section 3, we analyze and solve the subscription and pricing strategies of the different models. In Section 4, we show and discuss the results. Finally, in Section 5, we present the conclusions.

\section{Model Description}

2.1. General Model. This section models the following two sharing agreements:

(i) Pooling: the operators share equally the resource.

(ii) Priority sharing: the operators share the resource under different priorities.
In addition, we analyze in the Appendix the case where there is only one operator providing service; i.e., no priority is implemented and the operator behaves as a monopolist. This case will be used as a baseline for comparison in Section 4. A summary of the notation used in this paper is given in Table 1.

The network operator (aka. Operator 1) is the spectrum owner, while the virtual operator (aka. Operator 2) leases the resource under the specific sharing agreement. As part of the sharing agreement, the operator undertakes to communicate the data of the number of users to the other operator. We assume that each operator has its own subscriber base.

The network, i.e., the spectrum resource that is used for providing service, is modeled as an $\mathrm{M} / \mathrm{M} / 1 / \infty$ queue, where packets are generated by the subscribers of the two operators and are served according to the scheduling discipline described below. Operator $i$ has $n_{i}$ subscribers that independently generate packets following a Poisson process with a rate $\lambda_{d}$, so that for each operator we can define a Poisson packet arrival process with rate $\lambda_{i}=\lambda_{d} n_{i}$, and the sum of the two processes is also Poisson with rate $\lambda=\lambda_{1}+\lambda_{2}$. The service times of all packets are exponentially distributed with mean $1 / \mu$. We assume for stability reasons that $\lambda<\mu$.

Under the pooling agreement, the scheduling discipline is First-Come-First-Served and there is no priority. The mean packet system time $T_{i}$ can be computed as

$$
T_{1}=T_{2}=\left(\frac{1}{\mu-\left(n_{1}+n_{2}\right) \lambda_{d}}\right) .
$$

Under the priority sharing agreement, we propose that the service provided by the network be modeled by a Discriminatory Processor Sharing (DPS) discipline, where each customer has a relative priority and receives service at an instantaneous rate proportional to the priority [11, 27, 28]. A DPS queue basically works as follows: if there are $n$ customers with priorities $x_{1}, x_{2}, \ldots, x_{n}\left(x_{i} \leq 1\right.$ for $\left.i=1, \ldots, n\right)$, then the customer $i$ is served at a fraction $x_{i} / \sum_{j=1}^{n} x_{j}$ of the servers capacity [11]. In our analysis we use a DPS model with two relative priorities: $x_{1}=1-\gamma$ for the network operator and $x_{2}=\gamma$ for the virtual operator, where $x_{1}+x_{2}=1$ and $0 \leq \gamma \leq 1$. DPS is a mechanism much more flexible than priority queuing in order to model the sharing of a common resource, thanks to the $\gamma$ parameter. $T_{i}$ can be computed [11] as

$$
\begin{aligned}
& T_{1} \\
& =\frac{1}{\mu-\lambda_{d} n_{1}-\lambda_{d} n_{2}}\left(1+\frac{\lambda_{d} n_{2}(2 \gamma-1)}{\mu-(1-\gamma) \lambda_{d} n_{1}-\gamma \lambda_{d} n_{2}}\right), \\
& T_{2} \\
& =\frac{1}{\mu-\lambda_{d} n_{1}-\lambda_{d} n_{2}}\left(1-\frac{\lambda_{d} n_{1}(2 \gamma-1)}{\mu-(1-\gamma) \lambda_{d} n_{1}-\gamma \lambda_{d} n_{2}}\right) .
\end{aligned}
$$

Note that Preemptive Resume PQ, if the users were serviced in a processor sharing manner, is a special case of DPS discipline, since $\gamma=0$ gives strict priority to operator 1 users while $\gamma=1$ would give strict priority to operator 2 users [24]. 
TABLE 1: Summary of notations.

\begin{tabular}{|c|c|c|}
\hline & & Eq. \\
\hline \multicolumn{3}{|l|}{ General Model } \\
\hline Mean packet system time & $T_{i}$ & (1) \\
\hline Mean service rate & $\mu$ & (1) \\
\hline Subscribers mean arrival rate & $\lambda_{d}$ & (1) \\
\hline Operator l's number of users & $n_{1}$ & (1) \\
\hline Operator 2's number of users & $n_{2}$ & (1) \\
\hline Virtual Operator's relative priority & $\gamma$ & (2) \\
\hline Quality perceived by the users & $Q_{i}$ & $(4)$ \\
\hline Users sensitivity to delay & $\alpha$ & $(4)$ \\
\hline Conversion factor & $c$ & $(4)$ \\
\hline Users utility & $U_{i}$ & $(5)$ \\
\hline Price charged by operator $i$ & $p_{i}$ & $(5)$ \\
\hline Operator i's profits & $\Pi_{i}$ & $(6)$ \\
\hline \multicolumn{3}{|l|}{ Game Model } \\
\hline Operator 1's best response & $B R_{1}$ & (11) \\
\hline Operator 2's best response & $B R_{2}$ & (12) \\
\hline Operator 1's equilibrium price & $p_{1}^{*}$ & (13) \\
\hline Operator 2's equilibrium price & $p_{2}^{*}$ & (14) \\
\hline Operator l's equilibrium number of users & $n_{1}^{*}$ & (16) \\
\hline Operator 2's equilibrium number of users & $n_{2}^{*}$ & (16) \\
\hline Arbitrarily small positive value & $\epsilon$ & \\
\hline Boundary cases I-II & $\widehat{p}_{1 \gamma}$ & $(34)$ \\
\hline Boundary cases I-II & $\widehat{p}_{2 \gamma}$ & (35) \\
\hline Boundary cases III-IV & $\widehat{p}_{1 U}$ & $(36)$ \\
\hline Boundary cases II-IV & $\widehat{p}_{2 U}$ & $(37)$ \\
\hline \multicolumn{3}{|l|}{ The Appendix- Monopoly } \\
\hline Monopolistic operator's profits & $\Pi_{0}$ & (A.1) \\
\hline Monopolistic operator's number of users & $n_{0}$ & (A.1) \\
\hline Price charged by monopolistic operator & $p_{0}$ & (A.1) \\
\hline Users Utility & $U_{0}$ & (A.3) \\
\hline Monopolistic operator's optimal price & $p_{0}^{*}$ & (A.9) \\
\hline
\end{tabular}

We quantify the quality perceived by the users of each operator borrowing a variation of the expression used in $[23,25,29,30]$. In order to analyze the impact of the priority parameter $\gamma$, we assume that the users are homogeneous in their service time requirements for both operators.

$$
Q_{i} \equiv c T_{i}^{-\alpha}, \quad i=1,2,
$$

where $c>0$ is a conversion factor and $T_{i}$ has been defined and computed above. We define $0 \leq \alpha \leq 1$ as the user sensitivity to delay, since a greater $\alpha$ translates into a worse perceived quality, for a given delay. The user utility is proposed to be given by the difference between the quality perceived by the users in monetary units minus the price charged by the operator. Specifically, the user utility we model has the advantage of being interpretable in monetaryequivalent terms: since $c T_{i}^{\alpha}$ is the value that the outcome has to user $i$ and $p_{i}$ (in monetary units per second (m.u./s.)) is the price that the user pays, $U_{i}$ can be seen as the benefit (m.u./s.) of user $i$.

$$
U_{i} \equiv c T_{i}^{-\alpha}-p_{i}, \quad i=1,2 .
$$

We set the utility when users do not subscribe to the service to zero.

Note that a similar approach for modeling the user utility can be found at, e.g., [22, 31-33]. And this form of utility function can be related to the quasi-linear function widely used in microeconomic and telecommunications networks analysis as described in [34].

The network operator charges a price $p_{1}$ to its users, while the virtual operator charges a price $p_{2}$. We assume that no costs are incurred by the operators. The operators' profits will then be given by

$$
\Pi_{i}=\lambda_{d} n_{i}\left(p_{i}\right) p_{i}, \quad i=1,2 .
$$

Remark that the profits functions described in (6) should have been decreased by the operator's costs. Important 
components of these costs are the operating costs and the investment costs. We do not consider the operating costs, as their inclusion does not provide additional insight since they do not depend on the price of the service, while it makes the expression of profits less explicit. Related to the investment costs, we can consider them constant, given that the time scale on which the provider can adapt his network capacity $\mu$ is relatively long compared to the time scale on which prices vary $[25,30]$.

2.2. Game Model. From the above model description, we can observe the following strategic interactions:

(i) Flow i's subscription decision is influenced by operator i's pricing decision.

(ii) Operator i's profit depends on flow is subscription decision.

(iii) Flow i's subscription decision depends on flow j's decision with $(j \neq i)$, through the $Q_{i}$ factor.

(iv) Operator i's profit is then influenced by operator $j$ 's pricing decision, indirectly through flow $j$ 's subscription decision.

We conclude then that the above strategic scenario is amenable to game theory. Specifically, each operator will choose its pricing strategy $p_{i}$ aiming at maximizing its profit $\Pi_{i}$.

We will use a two-stage sequential game of the multileader follower kind, where, in the first stage, each operator chooses a price $p_{i}$ in order to maximize its profit $\Pi_{i}$. The game is solved using backward induction [35], which means that at Stage I players proceed strategically anticipating the solution of Stage II.

In the second stage, for each operator, the following applies. Each user takes its own subscription decision, trying to maximize the utility it gets from either subscribing to the operator or not. The user will observe the price published by the operator and will take the decision of service subscription. Assuming that number of users is high enough, the individual subscription decision of one user does not affect the utility of the rest of the users. Under these conditions, the equilibrium reached is that postulated by Wardrop [36]. Applying this notion of equilibrium, known as Wardrop equilibrium, to the user subscription problem, we postulate that

(i) either $n_{i} \geq 0$ and $U_{i}=0$

(ii) or $n_{i}=0$ and $U_{i}<0$.

Combining the above alternatives, we can identify four outcomes:

(i) Case I:

$$
\begin{aligned}
U_{1} & =0, \\
U_{2} & =0 \\
\text { and } n_{1} & \geq 0, \\
n_{2} & \geq 0
\end{aligned}
$$

(ii) Case II:

$$
\begin{aligned}
U_{1} & =0, \\
U_{2} & <0 \\
\text { and } n_{1} & \geq 0, \\
n_{2} & =0
\end{aligned}
$$

(iii) Case III:

$$
\begin{gathered}
U_{1}<0, \\
U_{2}=0 \\
n_{1}=0, \\
n_{2} \geq 0
\end{gathered}
$$$$
\text { and } n_{1}=0 \text {, }
$$

(iv) Case IV:

$$
\begin{aligned}
U_{1} & <0, \\
U_{2} & <0 \\
\text { and } n_{1} & =0, \\
n_{2} & =0
\end{aligned}
$$

In the first stage, each operator chooses a price in order to maximize its profits in a simultaneous and independent way. Each operator is not only aware of the users subscription decision in the second stage, but also of the rational behavior of the other operator. Under such circumstances, the equilibrium reached is the Nash equilibrium [37], where no player has an incentive to change its own strategy unilaterally.

The general method to discover the set of Nash equilibria is to obtain the best-response (BR) function of each operator and identify the crossing points [34]. The BR functions are defined as follows:

$$
\begin{aligned}
& B R_{1}\left(p_{2}\right)=\arg \max _{p_{1}} \Pi_{1}\left(p_{1}, p_{2}\right), \\
& B R_{2}\left(p_{1}\right)=\arg \max _{p_{2}} \Pi_{2}\left(p_{1}, p_{2}\right) .
\end{aligned}
$$

Once we have obtained the BR functions, we can obtain the set of Nash equilibria solving the following system of equations:

$$
\begin{aligned}
& p_{1}^{*}=\arg \max _{p_{1}} \Pi_{1}\left(p_{1}, p_{2}^{*}\right), \\
& p_{2}^{*}=\arg \max _{p_{2}} \Pi_{2}\left(p_{1}^{*}, p_{2}\right) .
\end{aligned}
$$

\section{Models Analysis}

In this section, we analyze the models described in Section 2.

3.1. Pooling. In this subsection, operator 1 and operator 2 are sharing the spectrum resource. The utility of the users can be obtained replacing (1) in (5).

$$
U_{i}=c \cdot\left(\mu-\left(n_{1}+n_{2}\right) \cdot \lambda_{d}\right)^{\alpha}-p_{i} \quad i=1,2
$$


We obtain the equilibrium values for $n_{1}$ and $n_{2}$, given $p_{1}$ and $p_{2}$, as follows.

(i) Case I: from (7) and (15) we obtain

$$
n_{1}^{*}+n_{2}^{*}=\frac{\left[\mu-\left(p_{1} / c\right)^{1 / \alpha}\right]}{\lambda_{d}}=\frac{\left[\mu-\left(p_{2} / c\right)^{1 / \alpha}\right]}{\lambda_{d}},
$$

which is only feasible if $p_{1}=p_{2}$. Without loss of generality, we can assume that $n_{1}^{*}=n_{2}^{*}$, so that

$$
\begin{aligned}
& n_{1}^{*}=\frac{\left[\mu-\left(p_{1} / c\right)^{1 / \alpha}\right]}{2 \lambda_{d}}, \\
& n_{2}^{*}=\frac{\left[\mu-\left(p_{2} / c\right)^{1 / \alpha}\right]}{2 \lambda_{d}} .
\end{aligned}
$$

(ii) Case II: from (8) and (15) we obtain

$$
\begin{aligned}
& n_{1}^{*}=\frac{\left[\mu-\left(p_{1} / c\right)^{1 / \alpha}\right]}{\lambda_{d}}, \\
& n_{2}^{*}=0, \\
& p_{1} \leq c u^{\alpha} . \\
& p_{2}>p_{1} .
\end{aligned}
$$

(iii) Case III: from (9) and (15) we obtain

$$
\begin{aligned}
& n_{1}^{*}=0, \\
& n_{2}^{*}=\frac{\left[\mu-\left(p_{2} / c\right)^{1 / \alpha}\right]}{\lambda_{d}}, \\
& p_{2} \leq c u^{\alpha}, \\
& p_{1}>p_{2} .
\end{aligned}
$$

(iv) Case IV: finally, from (10) and (15)

$$
\begin{aligned}
& n_{1}^{*}=0 \\
& n_{2}^{*}=0, \\
& p_{1}>c \mu^{\alpha}, \\
& p_{2}>c \mu^{\alpha} .
\end{aligned}
$$

As a corollary, price values for Case I comply with $p_{1} \leq$ $c \mu^{\alpha}$.

Table 2 summarizes the results of the users' subscription decision under a pooling agreement. Figure 1 shows a graphical representation of the regions for a specific configuration of the parameters.

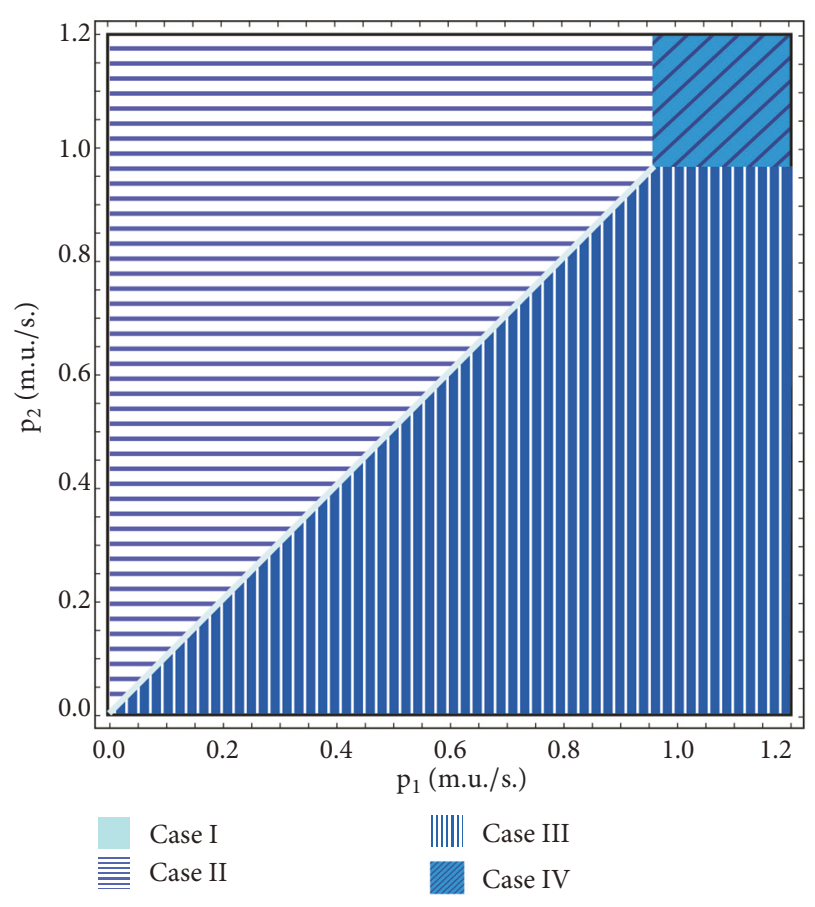

FIGURE 1: Wardrop equilibrium regions for pooling with $c=1, \alpha=$ $0.8, \mu=1$ packets per second $(\mathrm{p} / \mathrm{s}), \lambda_{d}=0.5 \mathrm{p} / \mathrm{s}$.

At this point we proceed to analyze the equilibrium prices $p_{i}^{*}$, given the values for $n_{1}^{*}$ and $n_{2}^{*}$. For this purpose we use (6) and we obtain the BRs as defined by (11) and (12):

$$
\begin{gathered}
B R_{1}\left(p_{2}\right)= \begin{cases}p_{1} \geq 0 & \text { if } p_{2}=0 \\
p_{2}-\epsilon & \text { if } 0<p_{2} \leq p_{0}^{*} \\
c \mu^{\alpha}\left(\frac{\alpha}{1+\alpha}\right)^{\alpha} & \text { if } p_{2}>p_{0}^{*}\end{cases} \\
B R_{2}\left(p_{1}\right)= \begin{cases}p_{2} \geq 0 & \text { if } p_{1}=0 \\
p_{1}-\epsilon & \text { if } 0<p_{1} \leq p_{0}^{*} \\
c \mu^{\alpha}\left(\frac{\alpha}{1+\alpha}\right)^{\alpha} & \text { if } p_{1}>p_{0}^{*}\end{cases}
\end{gathered}
$$

where $p_{0}^{*}$ is the profit maximizing price for a monopolistic operator, which is given by (A.9), and $\epsilon$ is an arbitrarily small positive value. The Nash equilibrium is the intersection of the BRs, which is easily derived as $p_{1}^{*}=0$ and $p_{2}^{*}=0$, with zero profits $\Pi_{1}^{*}=0, \Pi_{2}^{*}=0$. We conclude that a pooling agreement between a network operator and a virtual operator generates strategic interaction that drives prices and profits to zero.

3.2. Priority Sharing. In this section, we analyze the case where operator 1 and operator 2 share the spectrum resource according to a distribution of relative priority. As justified in 
TABLE 2: Users subscription decision-pooling.

\begin{tabular}{|c|c|c|c|c|}
\hline Case & $n_{1}^{*}$ & $n_{2}^{*}$ & $p_{1}$ & $p_{2}$ \\
\hline I & {$\left[\mu-\left(p_{1} / c\right)^{1 / \alpha}\right]$} & {$\left[\mu-\left(p_{2} / c\right)^{1 / \alpha}\right]$} & $p_{1} \leq c u^{\alpha}$ & $p_{2}=p_{1}$ \\
\hline & $2 \lambda_{d}$ & $2 \lambda_{d}$ & $P_{1}=c u$ & $P_{2}-P_{1}$ \\
\hline II & $\frac{\left[\mu-\left(p_{1} / c\right)^{-7}\right]}{\lambda_{d}}$ & 0 & $0 \leq p_{1} \leq c \mu^{\alpha}$ & $p_{2}>p_{1}$ \\
\hline III & 0 & $\frac{\left[\mu-\left(p_{2} / c\right)^{1 / \alpha}\right]}{\lambda_{d}}$ & $p_{1}>p_{2}$ & $0 \leq p_{2} \leq c \mu^{\alpha}$ \\
\hline IV & 0 & 0 & $p_{1}>c \mu^{\alpha}$ & $p_{2}>c \mu^{\alpha}$ \\
\hline
\end{tabular}

Section 2.1, this distribution is modeled by means of DPS and $T_{i}$ are now given by (2) and (3), so that the user utility will be

$$
\begin{aligned}
& U_{1} \\
& =c\left(\frac{\mu+(\gamma-1)\left(\lambda_{d} n_{1}+\lambda_{d} n_{2}\right)}{\left(\mu-\lambda_{d} n_{1}-\lambda_{d} n_{2}\right)\left(\mu-\gamma \lambda_{d} n_{2}+(\gamma-1) \lambda_{d} n_{1}\right)}\right)^{-\alpha} \\
& \quad-p_{1}, \\
& U_{2} \\
& =c\left(\frac{\mu-\gamma\left(\lambda_{d} n_{1}+\lambda_{d} n_{2}\right)}{\left(\mu-\lambda_{d} n_{1}-\lambda_{d} n_{2}\right)\left(\mu-\gamma \lambda_{d} n_{2}+(\gamma-1) \lambda_{d} n_{1}\right)}\right)^{-\alpha} \\
& \quad-p_{2} .
\end{aligned}
$$

Following a similar reasoning as in Section 3.1, we obtain the equilibrium values for $n_{1}^{*}$ and $n_{2}^{*}$ and the constraints on $p_{1}$ and $p_{2}$, which are shown in Table 3 . Figure 2 shows a graphical representation of the cases for a specific configuration of the parameters.

The following expressions for the cases boundaries apply:

$$
\begin{aligned}
& \widehat{p}_{1 \gamma}=c\left(\frac{\gamma \mu\left(p_{2} / c\right)^{-1 / \alpha}-\gamma+1}{-\gamma \mu+\gamma\left(p_{2} / c\right)^{1 / \alpha}+\mu}\right)^{-\alpha} \\
& \widehat{p}_{2 \gamma}=c\left(\frac{\left(p_{1} / c\right)^{-1 / \alpha}\left((\gamma-1) \mu-\gamma\left(p_{1} / c\right)^{1 / \alpha}\right)}{(\gamma-1)\left(p_{1} / c\right)^{1 / \alpha}-\gamma \mu}\right)^{-\alpha} \\
& \widehat{p}_{1 U}=c \mu^{\alpha} \\
& \widehat{p}_{2 U}=c \mu^{\alpha}
\end{aligned}
$$

Note that when $\gamma=1 / 2$, the priority sharing agreement reduces to the pooling agreement, since FCFS and DPS result in the same value for $T_{i}$.

Unlike Section 3.1, we have proceeded numerically in the analysis of Stage I, as regards the BRs and the Nash equilibrium.

\section{Results and Discussion}

In this section, numerical values are computed for the users, prices, and profits for the network and virtual operators under a priority sharing agreement. Note that the prices and the profits are zero for pooling, as concluded in Section 3.1.

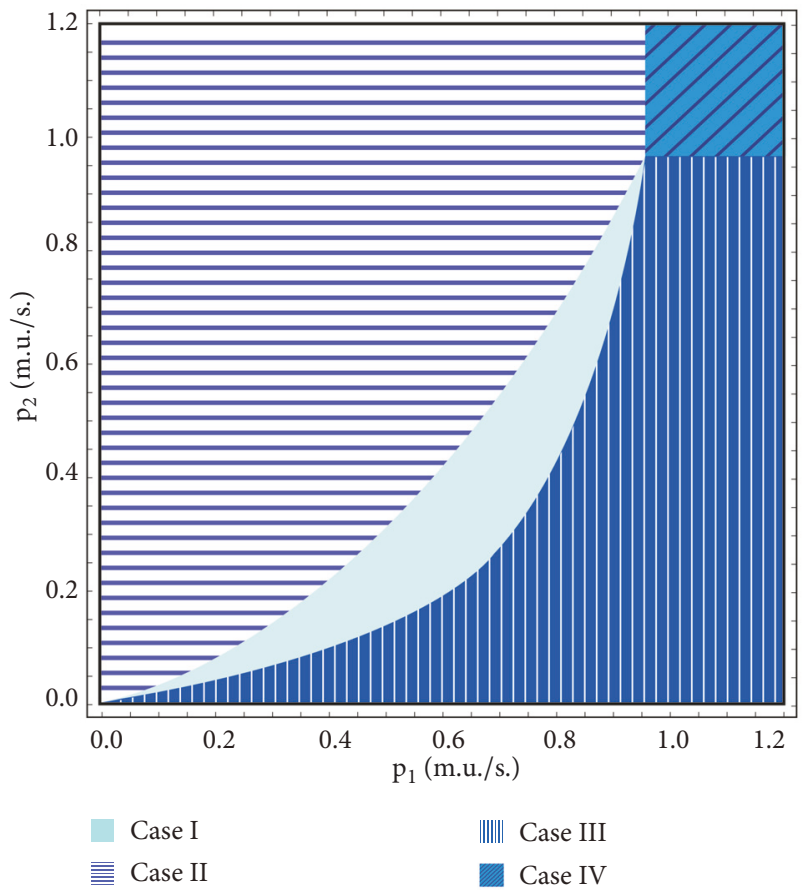

FIGURE 2: Wardrop equilibrium regions for priority sharing with $\gamma=$ $1 / 10, c=1, \alpha=0.8, \mu=1$ packets per second $(\mathrm{p} / \mathrm{s}), \lambda_{d}=0.5 \mathrm{p} / \mathrm{s}$.

We set the parameters $\alpha=\{0.5,0.8,1\}, c=1, \mu=1$ packet $/ \mathrm{s}, \lambda_{d}=0.01 \mathrm{packet} / \mathrm{s}$ and we vary $\gamma \in[0,1]$. Figures $3,4,5,6,7$, and 8 show the prices, the number of users, and profits, respectively. The values $p_{0}^{*}, n_{0}^{*}$, and $\Pi_{0}^{*}$ refer to the monopoly (the Appendix), while the values $p_{1}^{*}, n_{1}^{*}$, and $\Pi_{1}^{*}$ refer to the network operator, and the values $p_{2}^{*}, n_{2}^{*}$, and $\Pi_{2}^{*}$ refer to the virtual operator.

Figure 3 shows the effect of $\alpha$ and $\gamma$ (the virtual operator's relative priority) on the equilibrium prices of the monopolistic operator and operator 1 . The price $p_{1}$ decreases as $\alpha$ and $\gamma$ increase, since the QoS and priority $(1-\gamma)$ of the service that the network operator can provide to its subscribers are lower. However, when $\gamma$ approaches 1 the price $p_{1}$ increases. In this situation the network operator is giving strict priority to the virtual operator; i.e., the DPS discipline behaves as a PQ. This specific setting is analyzed in $[24,29,30]$ and the results match those shown in Figure 3. The monopolistic price $p_{0}^{*}$ is a constant value because it does not depend on $\gamma$ (Table 4). When $\gamma \in] 0,1\left[, p_{0}^{*}\right.$ is greater than the equilibrium prices, 
TABLE 3: Users subscription decision-priority sharing.

\begin{tabular}{|c|c|c|c|c|}
\hline Case & $n_{1}^{*}$ & $n_{2}^{*}$ & $p_{1}$ & $p_{2}$ \\
\hline & 1 & 1 & & \\
\hline $\mathbf{I}(\gamma \neq 1 / 2)$ & $\begin{array}{l}\lambda_{d}(\gamma-1)\left(c / p_{1}\right)^{1 / \alpha}+\gamma\left(c / p_{2}\right)^{1 / \alpha} \\
\mu \\
-\frac{1}{\mu} \frac{1}{1}\end{array}$ & $\begin{array}{l}\lambda_{d}(\gamma-1)\left(c / p_{2}\right)^{1 / \alpha}\left(c / p_{1}\right)^{-1 / \alpha}+\gamma \\
-\frac{1}{2} \frac{\gamma}{1}+\frac{1}{2}\end{array}$ & $p_{1} \leq \widehat{p}_{1 \gamma}$ & $p_{2} \leq \widehat{p}_{2 \gamma}$ \\
\hline $\mathbf{J}(x-1 / 2)$ & $\begin{array}{c}\overline{\lambda_{d}} \gamma\left(c / p_{2}\right)^{-1 / \alpha}\left(c / p_{1}\right)^{1 / \alpha}+\gamma-1 \\
{\left[\mu-\left(p_{1} / c\right)^{1 / \alpha}\right]}\end{array}$ & $\begin{array}{c}\overline{\lambda_{d}}(\gamma-1)\left(c / p_{1}\right)^{1 / \alpha}+\gamma\left(c / p_{2}\right)^{1 / \alpha} \\
{\left[\mu-\left(p_{2} / c\right)^{1 / \alpha}\right]}\end{array}$ & & \\
\hline $1(\gamma=1 / 2)$ & $2 \lambda_{d}$ & $2 \lambda_{d}$ & $p_{1} \leq p_{1 \gamma}$ & $p_{2}=p_{1}$ \\
\hline II & {$\left[\mu-\left(\frac{p_{1}}{c}\right)^{1 / n}\right]\left(\frac{1}{\lambda_{d}}\right)$} & 0 & $0 \leq p_{1} \leq \widehat{p}_{1 U}$ & $p_{2}>\widehat{p}_{2 \gamma}$ \\
\hline III & 0 & {$\left[\mu-\left(\frac{p_{2}}{c}\right)^{1 / \alpha}\right]\left(\frac{1}{\lambda_{d}}\right)$} & $p_{1}>\widehat{p}_{1 \gamma}$ & $0 \leq p_{2} \leq \widehat{p}_{2 U}$ \\
\hline IV & 0 & 0 & $p_{1}>\widehat{p}_{1 U}$ & $p_{2}>\widehat{p}_{2 U}$ \\
\hline
\end{tabular}

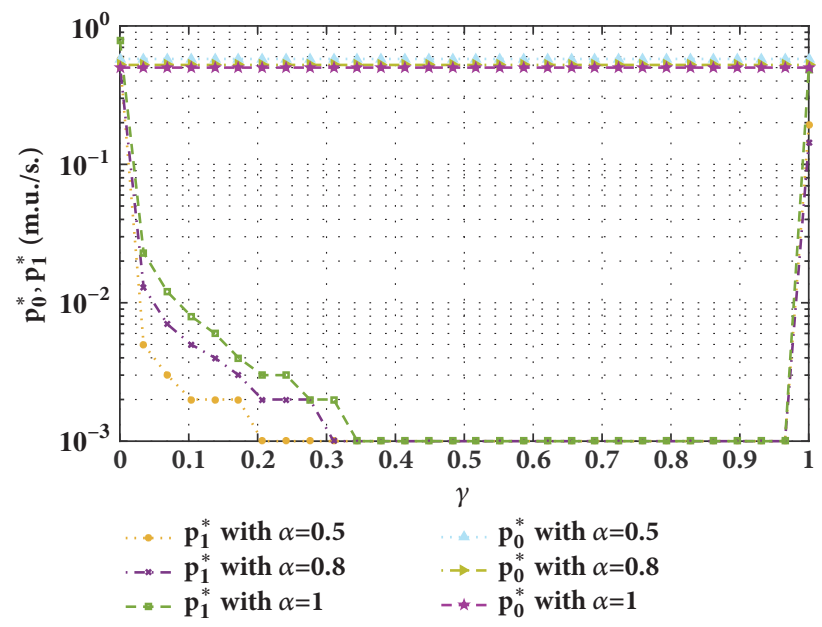

FIgURE 3: Prices of users for the network operator and the monopolistic operator as a function of $\gamma$ for different values of $\alpha$.

whereas $p_{i}^{*}=p_{0}^{*}$ when operator $i$ is given strict priority, since it receives a preemptive priority service; i.e., it is not affected by the nonpriority users.

Figure 4 shows the effect of $\alpha$ and $\gamma$ on the equilibrium prices of operator 2 . The prices $\left(p_{1}, p_{2}\right)$ behavior is symmetrical; i.e., $p_{2}$ exhibits the same behavior as the one described above but when $\alpha$ increases and $\gamma$ decreases from 1 to 0 .

We can conclude that the entry of the virtual operator causes the price charged both by the network operator and by the virtual operator to decrease; therefore, it is beneficial for the users. And we have numerically investigated that PQ is the limit case of DPS always providing the highest prices.

Figure 5 shows the effect of $\alpha$ and $\gamma$ on the number of users of operator $1 . n_{1}$ increases when $\alpha$ increases from 0 to 1 and $\gamma$ increases from 0 to 0.5 , which can be explained by the decrease in price $p_{1}$. At the same time, operator 2 loses all its users, and $n_{2}$ gets to zero. When $\gamma=0.5$, the system behaves as in the pooling agreement, i.e., zero prices and equal level of subscription. When $\gamma$ increases from 0.5 to 1 , the number of users switches and operator 1 loses its users. Figure 6 shows the effect of $\alpha$ and $\gamma$ on the number of users of operator 2 . The behavior of $n_{2}$ and $n_{1}$ is symmetrical; i.e., $n_{2}$ exhibits the same

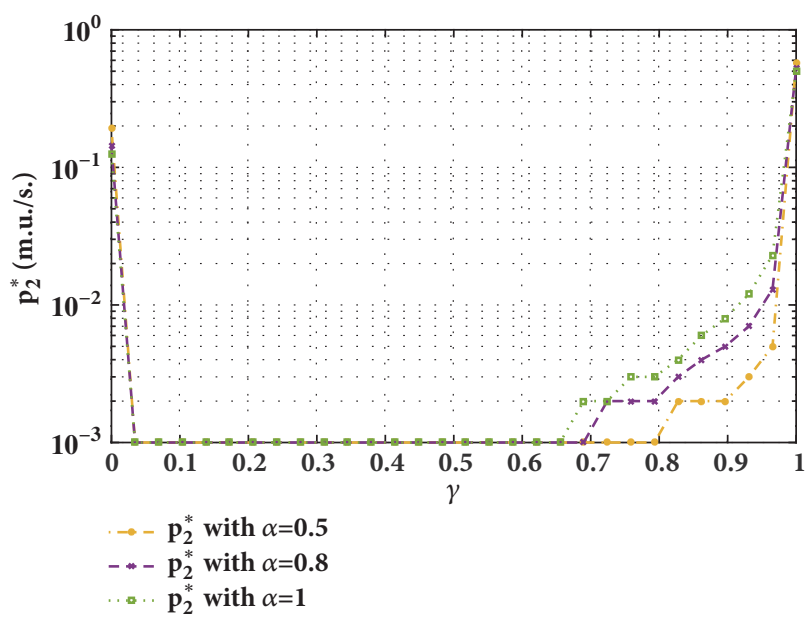

Figure 4: Prices of users of the virtual operator as a function of $\gamma$ for different values of $\alpha$.

behavior as the one described above but when $\alpha$ increases from 0 to 1 and $\gamma$ decreases from 1 to 0 .

The results show that the operator with the higher priority (e.g., operator 1 in the left hand side of Figure 5) is able to provide a satisfactory service to a maximum number of users, although the equilibrium price needs to be very low but nonnull (Figure 3). For $\gamma$ near the values of 0 and 1 , the number of users that the operator with the higher priority can serve is lower than the maximum; however, the equilibrium price is not so low as before, and the profits are greater (Figure 7 ). This specific setting is analyzed in $[24,29,30]$, where the discipline was Preemptive Resume Priority Queueing (which, if users are served in a processor-sharing manner, is equivalent to DPS for $\gamma=0$ or 1 ). The explanation for these results is related to the fact that only when $\gamma$ is near 0 or 1 , the QoS received by each user group is sufficiently different from each other so that the competition between the two operators is not too fierce and the prices need not be too low.

Figure 7 shows the equilibrium profit of each operator as $\alpha$ and $\gamma$ vary. We observe that the network operator suffers a significant reduction in its profit $\Pi_{1}$ when $\alpha$ and $\gamma$ increase between $0<\gamma<1$. The profits only recover when $\gamma$ approaches 
TABLE 4: Users, prices, and profit of one operator case.

\begin{tabular}{lccc}
\hline Case & $n_{0}^{*}$ & $p_{0}^{*}$ & $\Pi_{0}^{*}\left(p_{0}\right)$ \\
\hline I & {$\left[\mu-\left(\frac{p_{o}}{c}\right)^{1 / \alpha}\right]\left(\frac{1}{\lambda_{0}}\right)$} & $p_{0}^{*}=c \mu^{\alpha}\left(\frac{\alpha}{1+\alpha}\right)^{\alpha}$ & $\Pi_{0}\left(p_{0}^{*}\right)=c \mu^{\alpha+1}\left(\frac{\alpha}{1+\alpha}\right)^{\alpha+1}\left(\frac{1}{\alpha}\right)$ \\
II & 0 & $p_{0}>c \mu^{\alpha}$ & $\Pi_{0}\left(p_{0}^{*}\right)=0$ \\
\hline
\end{tabular}

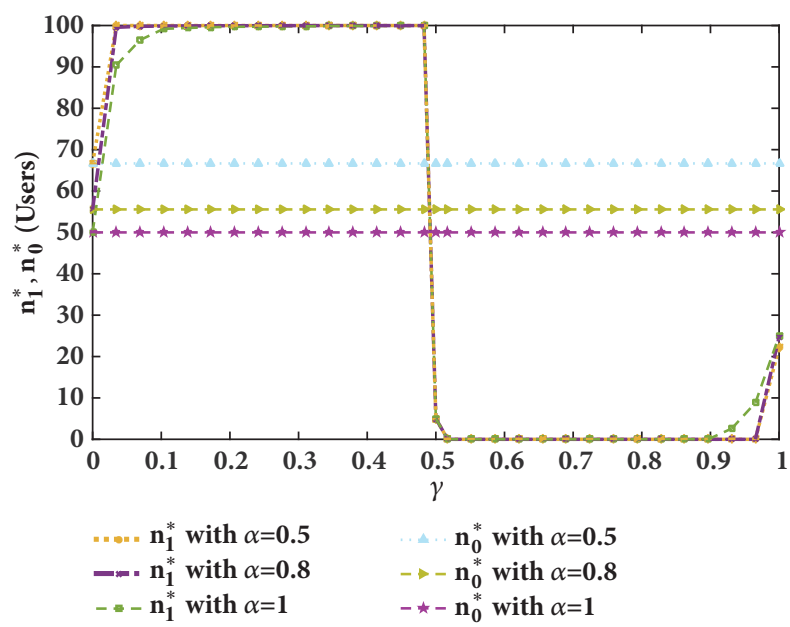

Figure 5: Number of users for the network operator and the monopolistic operator as a function of $\gamma$ for different values of $\alpha$.

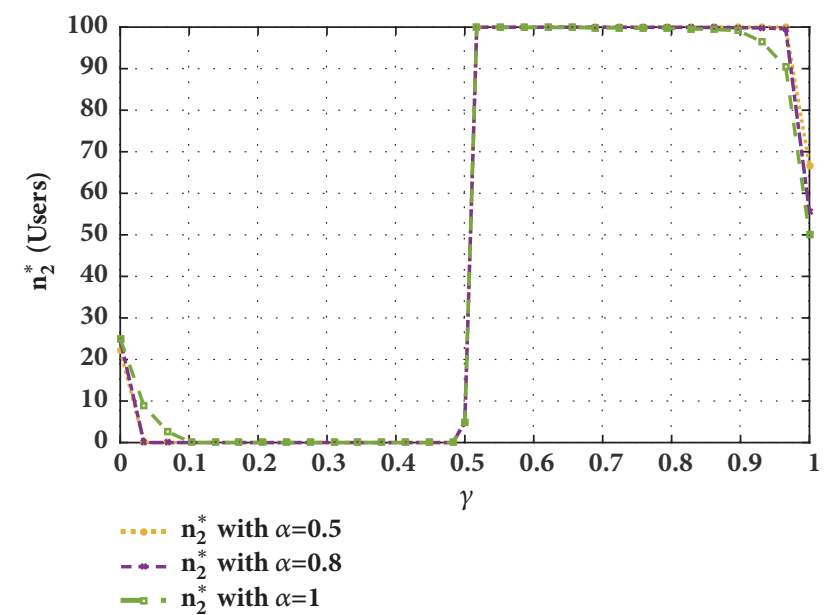

FIGURE 6: Number of users for the virtual operator as a function of $\gamma$ for different values of $\alpha$.

0 or 1; i.e., the DPS discipline behaves as a PQ. The behavior described is consistent with the combined behavior of the prices and the number of users, described above. The virtual operator behavior is symmetrical; i.e., $\Pi_{2}$ exibits the same behavior as the one described above but when $\alpha$ increases and $\gamma$ decreases from 1 to 0 .

When compared to the profit that the network operator would get in a monopoly, $\Pi_{0}$ (the Appendix), we conclude that the network operator is always worse off under a priority sharing agreement. Obviously, it is also worse off under a pooling agreement. However, we can determine a lump sum

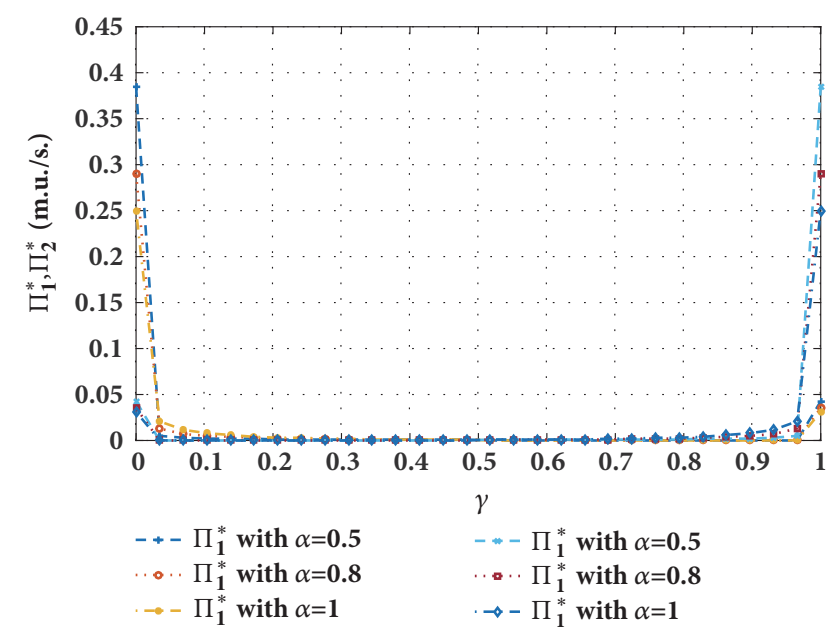

FIGURE 7: Profits for the network operator and the virtual operator as a function of $\gamma$ for different values of $\alpha$.

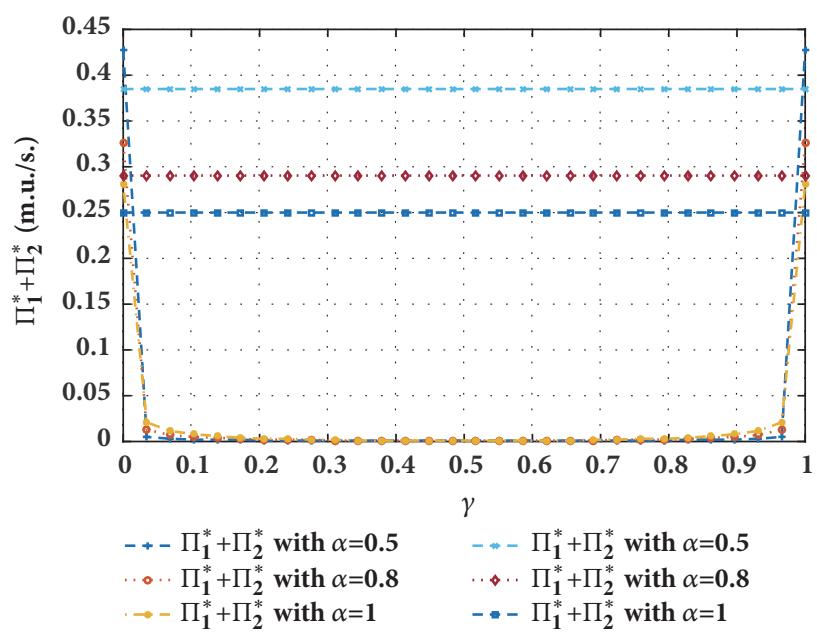

FIGURE 8: $\Pi_{1}^{*}+\Pi_{2}^{*}$ and profits for the monopolistic operator as a function of $\gamma$ for different values of $\alpha$.

payment $m$ that the virtual operator would make to the network operator in order to provide an incentive to the latter. Indeed, this payment should not only improve the situation of the network operator, that is, $\Pi_{1}+m \geq \Pi_{0}$, but also allow the virtual operator to obtain nonnegative profits $\Pi_{2}-m \geq 0$. Joining the two conditions we obtain $\Pi_{0}-\Pi_{1} \leq m \leq \Pi_{2}$. Thus, a necessary condition for the existence of a possible payment is $\Pi_{1}+\Pi_{2} \geq \Pi_{0}$. This condition obviously does not hold for the pooling agreement. As regards the priority 
sharing agreement, Figure 8 shows that this condition holds only for sufficiently small values of $\gamma$ or $1-\gamma$.

We can conclude that network sharing is incentive compatible under the following conditions: first, that a priority sharing agreement is reached and second, that either a very low or a very high relative priority is granted to the virtual operator willing to access the network operator resources.

\section{Conclusions}

In this paper, we have evaluated the issue of network sharing between a network operator and a virtual operator for profitmaximization purposes. We have studied the economic viability of the sharing under pooling and priority sharing agreements, and these are compared with a monopoly. Our main results suggest that the network operator is worse off under any circumstances under a pooling agreement. The entry of a virtual operator is desirable from the point of view of the users' prices. Additionally, for each parameter configuration there exist a range of values of $\gamma$, for which a lump sum payment can be designed so that the network operator has an incentive to let the virtual operator enter.

\section{Appendix}

\section{Monopoly}

In this appendix, we study the case where only the network operator provides the service to users. The first stage described in Section 2.2 is reduced to an optimal decision by the network operator in order to maximize its profits (A.1), whereas the second stage is reduced to two cases.

$$
\Pi_{0}=\lambda_{0} n_{0} p_{0}
$$

The utility in this case is

$$
U_{0}=c T_{0}^{-\alpha}-p_{0}
$$

where $T_{0}=\left(1 /\left(\mu-n_{0} \lambda_{0}\right)\right)$, and replacing $T_{0}$ in (A.2), we obtain

$$
U_{0}=c\left(\mu-n_{0} \lambda_{0}\right)^{\alpha}-p_{0}
$$

Analyzing the users' subscription decision we observe that given a price $p_{0}$ announced by the operator, the Wardrop equilibrium will be as follows.

(i) Case I: the number of users subscribing increases until the utility is zero. Therefore, the condition for this case is

$$
U_{0}=0
$$

Solving (A.3) under condition (A.4), the number of users is then

$$
n_{0}=\left[\mu-\left(\frac{p_{o}}{c}\right)^{1 / \alpha}\right]\left(\frac{1}{\lambda_{0}}\right)
$$

(ii) Case II: the price in (A.3) is so high that the utility is always negative. Therefore the condition for this case is

$$
U_{0}<0
$$

Under condition (A.6), the users do not subscribe the service. Therefore, the number of users is

$$
n_{0}=0
$$

Assuming equilibrium is Case I, we can obtain the profits replacing (A.5) in (A.1).

$$
\Pi_{0}=\left[\mu-\left(\frac{p_{0}}{c}\right)^{1 / \alpha}\right] p_{0}
$$

We can maximize the profit setting its derivative with respect to the price $\left(p_{0}\right)$ equal to zero; the result of $\left(p_{0}^{*}\right)$ is

$$
p_{0}^{*}=c \mu^{\alpha}\left(\frac{\alpha}{1+\alpha}\right)^{\alpha}
$$

Finally we can obtain the maximum profit replacing (A.9) in (A.1).

$$
\Pi_{0}\left(p_{0}^{*}\right)=c \mu^{\alpha+1}\left(\frac{\alpha}{1+\alpha}\right)^{\alpha+1}\left(\frac{1}{\alpha}\right)
$$

Table 4 shows the results of the prices and profit for this case.

\section{Data Availability}

No data were used to support this study.

\section{Conflicts of Interest}

The authors declare that there are no conflicts of interest regarding the publication of this article.

\section{Acknowledgments}

This work was supported by the Spanish Ministry of Economy and Competitiveness through projects TIN2013-47272-C2-1R (cosupported by the European Social Fund) and BES-2014068998 and partially supported by the Salesian Polytechnic University of Ecuador through a Ph.D. scholarship granted to the first author.

\section{References}

[1] V. Tikhvinskiy and G. Bochechka, Conceptual aspects of $5 \mathrm{G}$ construction, 2013.

[2] X. Zhang, W. Cheng, and H. Zhang, "Heterogeneous statistical QoS provisioning over 5G mobile wireless networks," IEEE Network, vol. 28, no. 6, pp. 46-53, 2014.

[3] W. Cheng, X. Zhang, and H. Zhang, "Heterogeneous statistical QoS provisioning over 5G wireless full-duplex networks," in Proceedings of the IEEE INFOCOM 2015 - IEEE Conference on Computer Communications, pp. 55-63, Kowloon, Hong Kong, April 2015. 
[4] P. Marsch, I. Da Silva, O. Bulakci et al., "5G Radio Access Network Architecture: Design Guidelines and Key Considerations," IEEE Communications Magazine, vol. 54, no. 11, pp. 24-32, 2016.

[5] A. Kliks, B. Musznicki, K. Kowalik, and P. Kryszkiewicz, "Perspectives for resource sharing in $5 \mathrm{G}$ networks," Telecommunication Systems, vol. 68, no. 4, pp. 605-619, 2018.

[6] K. Samdanis, X. Costa-Perez, and V. Sciancalepore, "From network sharing to multi-tenancy: The $5 \mathrm{G}$ network slice broker," IEEE Communications Magazine, vol. 54, no. 7, pp. 32-39, 2016.

[7] M. Andrews, M. Bradonjić, and I. Saniee, "Quantifying the benefits of infrastructure sharing," in Proceedings of the the 12th workshop, pp. 1-6, Cambridge, Massachusetts, June 2017.

[8] N. Alliance, " $5 g$ white paper," Next generation mobile networks, white paper, 2015.

[9] J. G. Andrews, S. Buzzi, and W. Choi, "What will 5G be?" IEEE Journal on Selected Areas in Communications, vol. 32, no. 6, pp. 1065-1082, 2014.

[10] N. Chee-Hock and S. Boon-Hee, "Queueing Modelling Fundamentals: With Applications in Communication Networks: Second Edition," Queueing Modelling Fundamentals: With Applications in Communication Networks: Second Edition, pp. 1-271, 2008.

[11] R. Hassin and M. Haviv, To queue or not to queue: Equilibrium behavior in queueing systems, vol. 59, Springer Science \&amp; Business Media, 2003.

[12] J. C. Harsanyi and R. Selten, A general theory of equilibrium selection in games, MIT Press, Cambridge, MA, 1988.

[13] S. Lasaulce, M. Debbah, and E. Altman, "Methodologies for analyzing equilibria in wireless games: A look at pure, mixed, and correlated equilibria," IEEE Signal Processing Magazine, vol. 26, no. 5, pp. 41-52, 2009.

[14] P. Di Francesco, F. Malandrino, T. K. Forde, and L. A. DaSilva, "A Sharing- and Competition-Aware Framework for Cellular Network Evolution Planning," IEEE Transactions on Cognitive Communications and Networking, vol. 1, no. 2, pp. 230-243, 2015.

[15] P. Caballero, A. Banchs, G. De Veciana, and X. Costa-Perez, "Network slicing games: Enabling customization in multitenant networks," in Proceedings of the 2017 IEEE Conference on Computer Communications, INFOCOM 2017, USA, May 2017.

[16] L. Cano, A. Capone, G. Carello, M. Cesana, and M. Passacantando, "Cooperative Infrastructure and Spectrum Sharing in Heterogeneous Mobile Networks," IEEE Journal on Selected Areas in Communications, vol. 34, no. 10, pp. 2617-2629, 2016.

[17] S. DOro, F. Restuccia, T. Melodia, and S. Palazzo, "LowComplexity Distributed Radio Access Network Slicing: Algorithms and Experimental Results," https://arxiv.org/abs/1803 .07586 .

[18] S.-L. Hew and L. B. White, "Cooperative resource allocation games in shared networks: Symmetric and asymmetric fair bargaining models," IEEE Transactions on Wireless Communications, vol. 7, no. 11, pp. 4166-4175, 2008.

[19] F. Teng, D. Guo, and M. L. Honig, "Sharing of Unlicensed Spectrum by Strategic Operators," IEEE Journal on Selected Areas in Communications, vol. 35, no. 3, pp. 668-679, 2017.

[20] Y.-T. Lin, H. Tembine, and K.-C. Chen, "Inter-operator spectrum sharing in future cellular systems," in Proceedings of the 2012 IEEE Global Communications Conference, GLOBECOM 2012, pp. 2597-2602, USA, December 2012.

[21] Y. Zhu, G. Meng, Y. Qiu, Z. Ji, G. Xie, and Y. Song, "Spectrum Sharing for Mobile Virtual Networks: A Game Theoretic
Approach," in Proceedings of the 2018 13th APCA International Conference on Automatic Control and Soft Computing (CONTROLO), pp. 179-183, Ponta Delgada, Portugal, June 2018.

[22] H. Mendelson, "Pricing computer services: Queueing effects," Communications of the ACM, vol. 28, no. 3, pp. 312-321, 1985.

[23] Y. Hayel, D. Ros, and B. Tuffin, "Less-than-best-effort services: pricing and scheduling," in Proceedings of the IEEE INFOCOM 2004, pp. 66-75, Hong Kong, PR China.

[24] Y. Hayel and B. Tuffin, "Pricing for Heterogeneous Services at a Discriminatory Processor Sharing Queue," in NETWORKING 2005. Networking Technologies, Services, and Protocols; Performance of Computer and Communication Networks; Mobile and Wireless Communications Systems, vol. 3462 of Lecture Notes in Computer Science, pp. 816-827, Springer Berlin Heidelberg, Berlin, Heidelberg, 2005.

[25] M. Mandjes, "Pricing strategies under heterogeneous service requirements," Computer Networks, vol. 42, no. 2, pp. 231-249, 2003.

[26] J. Markendahl, Mobile network operators and cooperation: A teleeconomic study of infrastructure sharing and mobile payment services [Ph.D. dissertation], KTH, 2011.

[27] L. Kleinrock, "Time-shared systems: a theoretical treatment," Journal of the ACM, vol. 14, no. 2, pp. 242-261, 1967.

[28] E. Altman, K. Avrachenkov, and U. Ayesta, "A survey on discriminatory processor sharing," Queueing Systems, vol. 53, no. 1-2, pp. 53-63, 2006.

[29] L. Guijarro, V. Pla, and B. Tuffin, "Entry game under opportunistic access in cognitive radio networks: A priority queue model," in Proceedings of the 6th IFIP/IEEE Wireless Days Conference, WD 2013, Spain, November 2013.

[30] A. Sanchis-Cano, L. Guijarro, V. Pla, and J. R. Vidal, "Economic viability of HTC and MTC service provision on a common network infrastructure," in Proceedings of the 14th IEEE Annual Consumer Communications and Networking Conference, CCNC 2017, pp. 1044-1050, USA, January 2017.

[31] P. Vamvakas, E. E. Tsiropoulou, and S. Papavassiliou, "Dynamic Provider Selection \& Power Resource Management in Competitive Wireless Communication Markets," Mobile Networks and Applications, vol. 23, no. 1, pp. 86-99, 2018.

[32] E. Altman, T. Boulogne, R. El-Azouzi, T. Jiménez, and L. Wynter, "A survey on networking games in telecommunications," Computers \& Operations Research, vol. 33, no. 2, pp. 286-311, 2006.

[33] P. Belleflamme and M. Peitz, Industrial organization: markets and strategies, Cambridge University Press, 2015.

[34] P. Maillé and B. Tuffin, Telecommunication Network Economics: from Theory to Applications, Cambridge University Press, Cambridge, UK, 2014.

[35] E. N. Barron, Game Theory: An Introduction, John Wiley \& Sons, 2013.

[36] J. G. Wardrop, "Some theoretical aspects of road traffic research," in Proceedings of the Inst Civil Engineers, London, UK.

[37] H. Varian, "Intermediate Microeconomics: A Modern Approach," in Proceedings of the Ninth International Student Edition, 2014. 


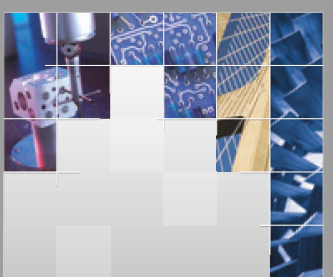

\section{Enfincering}
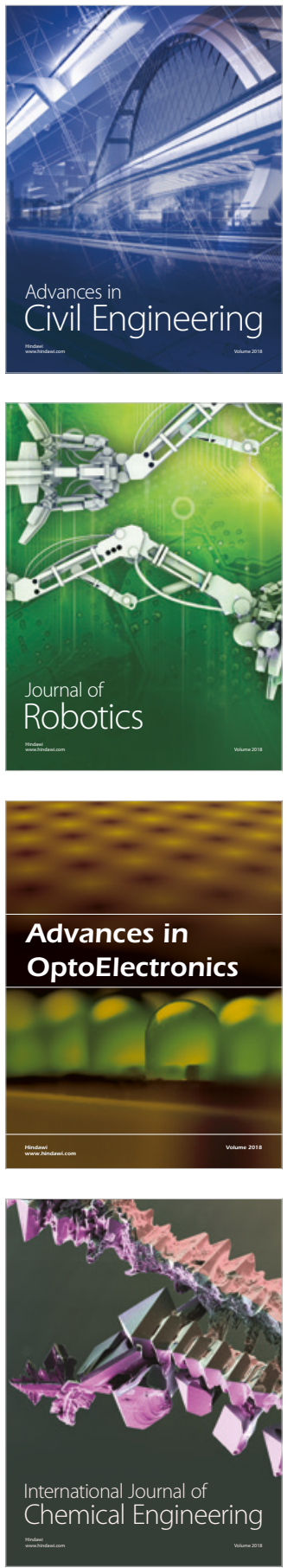

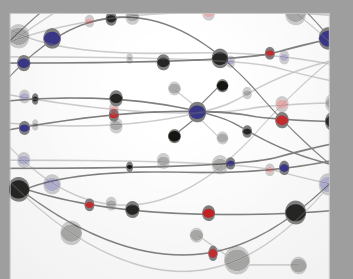

\section{Rotating \\ Machinery}

The Scientific World Journal

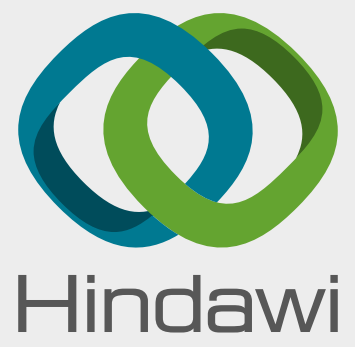

Submit your manuscripts at

www.hindawi.com
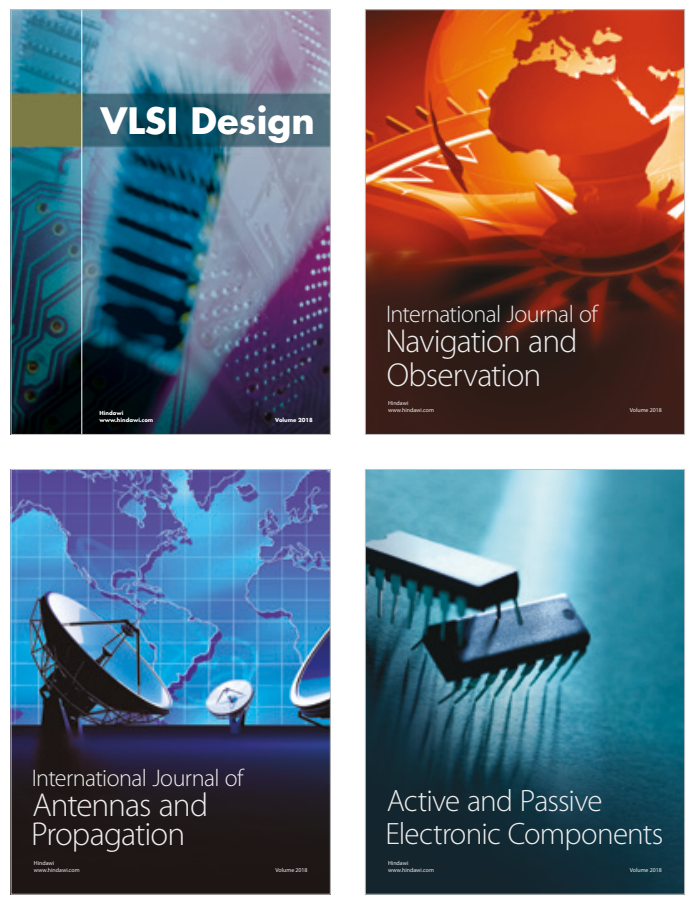
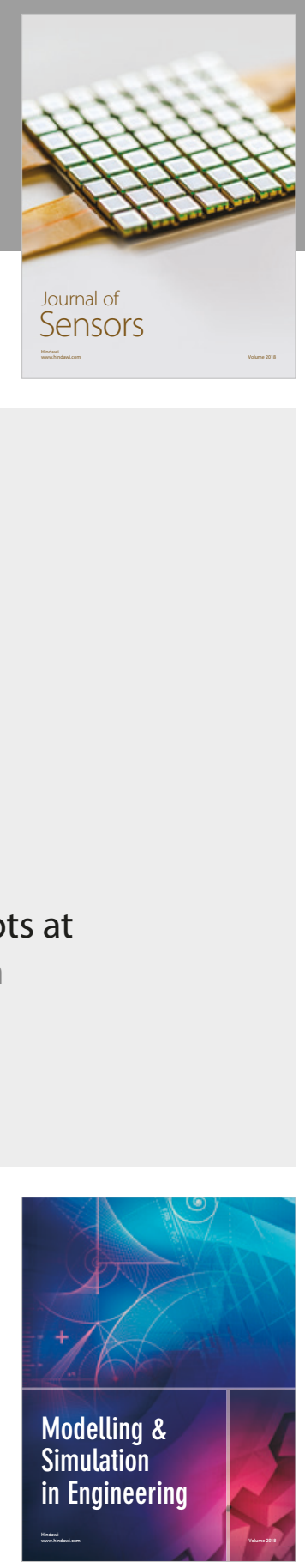

\section{Advances \\ Multimedia}
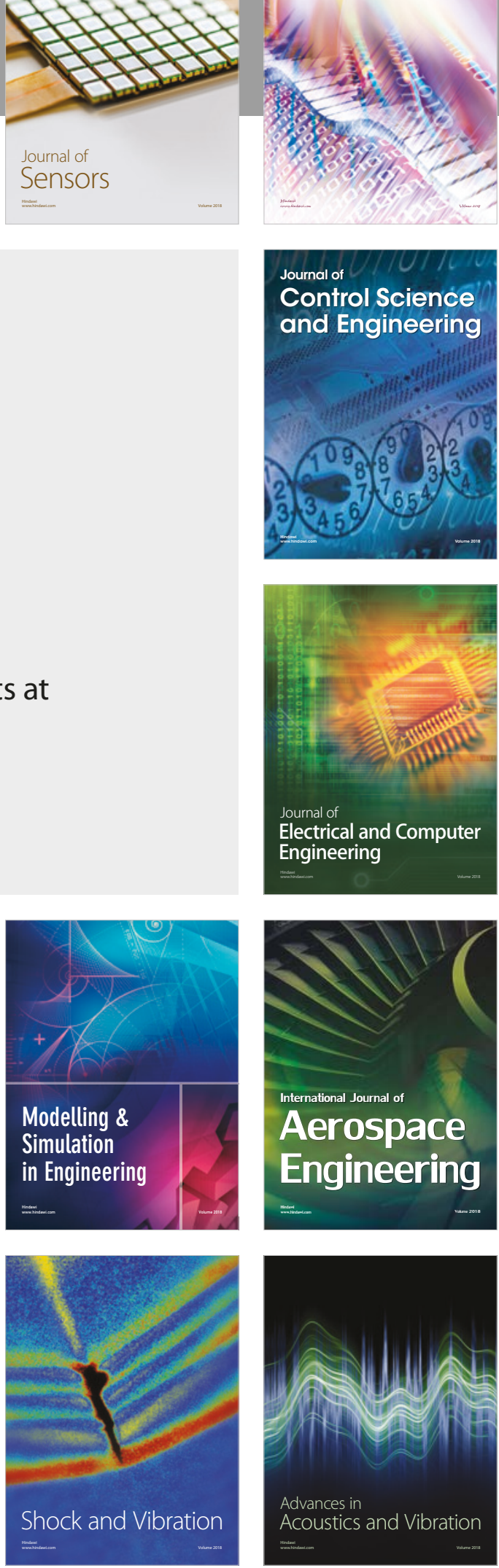